التحولات المفاهيميه للفن التشكيلي السعودي المعاصر

\title{
Conceptual transformations of contemporary
}

\author{
Saudi art
}

$$
\begin{aligned}
& \text { إعداد } \\
& \text { د. ابتسام بنت سعود الرشيد } \\
& \text { د. شذا بنت إبراهيم الاصقه }
\end{aligned}
$$

جامعة الملك سعود

كلية التربية قسم التربية الفنية

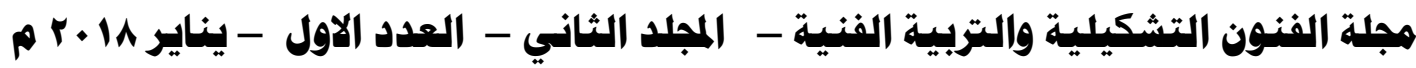




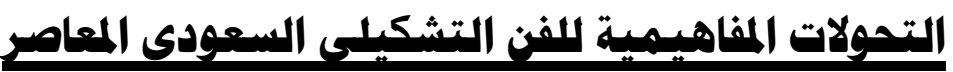

هبلة الفنون التشكيلية والتزبية الفنية - الملد الثاني - العدد الاول - يناير ما م م م

$1 \leq 1$ 
المقدمة:

كان التخلي عن المفاهيم التقليدية المنبعة في الفنون وتخطي مجالات الفن من اجل رؤية جديدة للواقع والتوجه نحو العمل بمادة العالم بشكل مباشر؛ من أهم أسباب ظهور الفن المفاهمي. الذي أعتمد على الأساس الفكري وأساليب مغايره للتعبير عن الفكرة المحددة، فهذا النوع من الفن حسي يتضمن كل العمليات الفكرية، ويتحرر من المهارة الحرفية للفنان حيث تصبح الفكرة هي الهذف الحقبقي للفن بدلا من جمالية العمل الفني فقط، بحيثأعتد على بقاء الأثر الفني في نفوس المتذوقين.

لقد تخطى المضمون المفاهيمي قواعد الفن القديم من اجلروئية جديدة للواقع. فالواقع هنا هو المجال الاساسي للأدراك الجمالي ادراكا فنباجديدا ومدلول (الفن المفاهيمي) هو التبدل الكلي في العلاقات التقليدية في العمل الفنبيين الفكرة والتعبير. وعلى العمل أن يصدر مشكلةعقلية بصرف النظر عن القيم الصادرة منه. حيث يتشكل العمل الفني بواقع أسئلة تفترض تأثيرها في زمن العرض ومدة الاعداد له. حيث تتضافر التقنية والفضاء الملائم

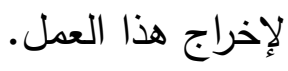

ويتميز هذا النوع من الفن بكونه أكثر إنسانية وله وظيفة اجتماعية وتعليمية، لأنه يعطي المشاهد المعلومات للبحث والتتقيب عن المفاهيم والافكار، وتختلف عن طبيعة الفن البصري المورفولوجي الذي يقدم شيئا جميلا أو قييحا بصريا فقط. فالفن الدفاهيمي يحقق

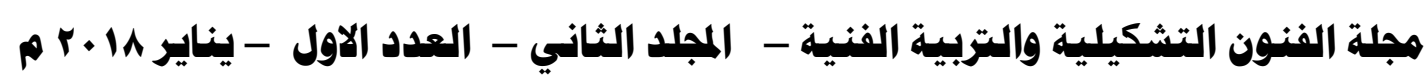




\section{التحولات المفاهيسيةية للفن التشكيلي السعودى المعاصر}

المفهوم الفني الذي تكلم عنه جوزيف كوزوث (Joseph Cosuth) غير موجود في الأثنياء الثانوية، أما الفن فهو موجود في مفهوم الفنان عن العمل الفني، أي يجب طرح القضية الفنية التتكيلية مثلما تطرح قضايا فكرية، اجتماعية، علمية أو أية قضايا إبداعية أخرى .

وبهذا المفهوم المتميز أنتشر الفن الدفاهيمي في جميع أرجاء العالم بمختلف مجالاته منها فن الميديا، والفن الرقمي، وفن الفيديو، وفن التصوير وفن الفراغ... ومن أهم المناطق التي تأثرت في مفهوم الفن المفاهيمي هي المملكة العربية السعودية حيث أعتمد فنانيها الففهوم المفاهيمي بشكل مبسط لا يمكن ملاحظته في بادئ الامر ،ولكن مع الاندماج الحضاري والفكري أعتمد الكثير من الفنانين التشكيلينالسعوديين المعاصرين المفهوم المفاهيمي في أعمالهم الفنية بشكل ملحوظ مرتبطين بالفكرة الأساسية من العمل الفني ومطوعين الخامات والتقنيات لتحقيق الهدف منها.

لقد مر الفن المفاهيمي في الفن التشكيلي السعودي المعاصربالعديد من التحولات الفكرية والتقافية والفلسفية والاجتماعية حتى وصل الى طابع مميز عالمي في الابداع الفني. فالفن التتكيلي المعاصرالسعودي هو نتاج نمو فكري وتقني ساهم في تحول الثكل الفني لخدمة فكر الفنان ووعيه بقضايا المجتمع والحياة النقافية والسياسية (قاسم، I 1 •rم).

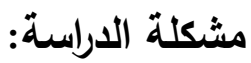
ومما سبق يمكن تحديد مشكلة البحث في التساؤل التالي: 
التحولات المفاهيسيمية للفن التشكيلي السعودى المعاصي

• ما التحولات المفاهيميه للفن التشكيلي السعودي المعاصر؟.

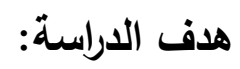

تهدف الدراسة الى :

التعرف على المضمون المفاهيمي في الفنون التشكيلية المعاصرة.

• الكثف عن التحولات المفاهمية في الفنون التتكيلة لسعودي المعاصرة.

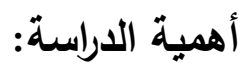

الأهمية النظرية:

• القاء الضوء على التحولات المفاهيميهللفنون التشكيلية السعودية المعاصرة.

الأهمبة التطبيقية:

• دراسة وتحليل التحولات المفاهيمية لفن التصوير التتكيلي السعودي.

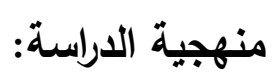

يتبع البحث الحالي المنهج الوصفي التحليلي في وصف مفهوم الفن المفاهيمي، وتحليل نماذج من الاعمال الدفاهيمية السعودية.

حدود البحث:

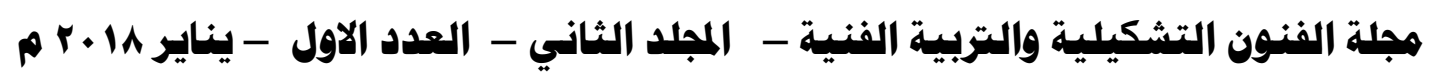
101 


\section{التحولات المفاهيسية للفن التشكيدي السعودى المعاصر}

الحدود الموضوعية : الفن المفاهيمي

الحدود المكانية: الفنون المعاصرة في المملكة العربية السعودية.

الحدود الزمانية: نماذج من الفن المفاهيمي التتكيلي السعودي المعاصر من عام (.... مج)

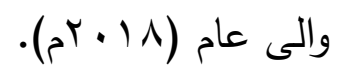

المصطلحات:

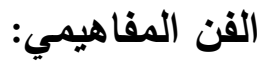

مدلول الفن المفاهيمي هو التبادل الكلي للعلاقات التقليدية في العمل الفني بين

الفكرة والتعبير، وعلى العمل الفني اصدار مشكلة عقلية بصرف النظر عن القيم الصادرة

منه، بحيث يتشكل العمل الفني من خلال التساؤلات التي بطرحها الفنان على المتلقي داعية

إياه الى التأمل العقلي للوصول الى فكرة العمل الفني ويكون ذلك بتضافر التنتية والخامات والأساليب الفنية المستخدمة في العمل مع فضاء ومكان العرض (أمهز،999 م).

ويعتمد هذا البحث على أن الفن المفاهيمي هو التشكيلات الفنبة المتتوعة التي

يقدمها الفنان للمنلقي بما تحمله من مضامين فكرية يمكن الوصول اليها بتضافر التأمل الفكري مع التتكيلات التعبيرية للعمل نفسه.

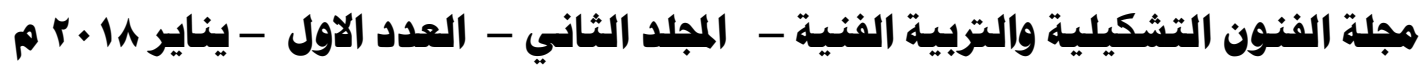


الفن التشكيلي المعاصر : n

أن المقصود بالفنون النتكيلة " هي تلك الفنون التعبيرية التي تحمل مضامين فكرية

وجمالية ولا تمت بصله لأغراض الاستعمالات اليومية، ويتم إدراكها وتذوقها وتقديرها عن

طريق حاسة البصر أو الروئة البصرية المباشرة، ونسمى بالفنون البصرية"(النصار ، ـ • ؟،

ص9 ب ()، وهو الفن الذب يرتبط بمتغيرات المجتمع المعاصر الاقتصادية والثقافية والعلمية،

$$
\text { و يشمل جميع الوسائط المستخدمة في التعبير عن الفكرة (قاسم، (1 • rم). }
$$

ويمكن تعريف الفن التشكيلي المعاصر في هذا البحث بأنه التتكيلات الفنية

التعبيرية والتي تحمل مضامين الفكر الفلسفي والايديولوجي والتقافي والاجتماعي والديني

للحضارة والمجتمع الذي يتتبعه وندرك بواسطة حاسة الابصار .

المحور الأول: المضمون المفاهيمي في الفنونالتثكيلية/لمعاصرة.

يعد الفن المفاهيمي من الفنون التي جذبت العديد من الفنانين في العصر الحديث لما

يحمله من مضمون فلسفي فكري. فقدأهتم الفنان الدفاهيمي بمجال واسع من المعلومات والموضوعات التي لا يمكن جمعها في شيء واحد بسهوله،فالفن المفاهيمي يعتمد على مضمون الفكرة المراد التعبير عنها كهذف أساسي في التعبير الفني. وتخلف أسسه التتكيلية والتعبيرية عن غيره من الفنون، ويقول محسن عطية عن الفنون المعاصرة ومنها الفن المفاهيمي انها ابتعدت عن الوحدة الميتافزيقية متجه الى فكرة العمل المتشكل بالانفصال والتقطع مما يدعو المتلقي

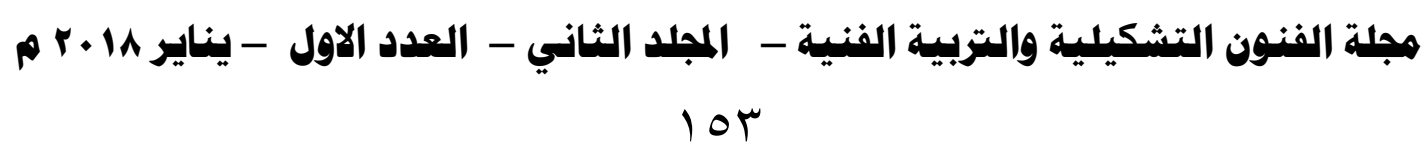




\section{التحولات المفاهيسية للفن التشكيلِي السعودى المعاصر}

للروية التفكيكية للعمل الفني موظفا التعارض الفني للتعبير جماليات التشكيلات المنفتحة (11 • (Y). حيث يمكن أن يستخدم الفنان نص يشرح العمل كما يمكنه توجيهه المتلقي لهدف العمل الفني بصورة أفضل عن طريق المقترحات المكتوبة والصور الفتوغرافية والوثائق والخرائط والرسوم البيانية والفيديو وأجسام الفنانين أنفسه؛ ليتمكن المتذوق من استخراج الفكرة نفسها من

$$
\text { التشكيل الفني (الحاتمي،با • rم). }
$$

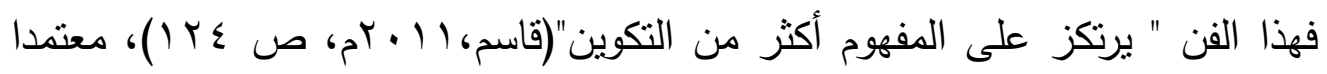
على الفراغ والعلاقات بين المساحات والتقنيات والأساليب بجعلها المادة الاساسية في الموضوع. فتحول الفن في المضمون الدفاهيمي منشيء مجمد ماديا إلى وسيلة استعلام وبحث والتساؤلات

$$
\text { يفترضها المتلقي للعمل في زمن العرض (الحاتمي، ا ـ بم). }
$$

أن هذا النوع من الفنون عمد على تأكيد مبدأ الهدف والفكرة الأساسية لتنكبل العمل

الفني ويؤكد ذلك سلويت "Sollewitt "مشيرا بأن المفهوم هو الجانبالأكثر أهمية في العمل الفني المفاهيمي ويضيف بأن الفنان مفكر ومبدع أكثر منه حرفي في المجال المفاهيمي بالفنون، وأن المتلقين يساهمون في تحقيق الفكرة الفنبة من العمل.

والفن المفاهيمي يعتبر فن حدسي بحسب رؤية د كتور محسن" لأنه يتضمن كل العمليات الفكرية لتكون الفكرة وهي الهُف الحقيقي من الفن بدلا من الأثر الفني"(؟ ...؟، صع V). أن هذالالمفهوم يجتاز تجربة الواقع ويقع بين تجربته لهذا الواقع وهما الثكل 


\section{التحولات المفاهيسية للفن التشكيلِي السعودى المعاصر}

والمضمون.فالفن المفاهيمي من النوع الذي يدمج الفن بالحياة بشكل مباشر ودون وسائل اتصال. ويعد هذا النوع من الفنون التثكيلية ذات مدلول مادي للعمل الفني، وتبادل كلي في العلاقات التقليدية من الموضوع والتعبير، لتكون الفكرة هي الهدف الفعلي للتشكيل الفني(أمهز،777 (7)م. فقد قلب الفن المفاهيمي أساليب التصوير والتتكيل الفني بتفكيك شرعية العمل، فأصبح العمل الفني يتحقق بالمشادة وتذوق عناصره (عطية، I| + آم). كما يرى الدكتور صالح رضا أن الفنون المفاهيك المعاصرة أهتىت " بتأكيد الفاعلية التعبيرية لماده العمل الفني واستخدام مضامين جديده في معالجة الواقع المرئي، وذلك من خلال الكيفيات الاسلوبية في عمليات التصوير الفني قدمت للششاهد فرصة الاتصال بنشاط الفنان وبتصوراته الجمالية"(ه . ب rم ،ص ^9)، وهذا ما أعتمده الدفهوم الدفاهيمي في الفنون التشكيلية متجها الى التجريد والتحوير والحذف والاضافة في عناصر العمل الفني، مفصحا بلغة الألوان والاشكال والاحجام والظل والنور عن القيمة الفنية

$$
\text { للفكرة المعبر عنها(نفادي،م . - rم). }
$$

أصبح الفن التشكيلي تصوريا تفاعليا، معطيا للمنذوق الدور الفاعل في الجمالية التعبيرية، موكدا على أهمية الوعي لوظيفة للثكل في التتكيل الفني المعاصر بكونه نظاما دلالياً

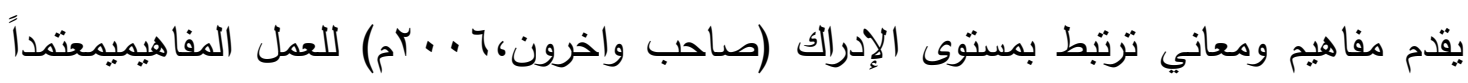
على الاستعارة التعبيرية لتقديم عمل حدسي يتطلب التفكير المتعقف(الحاتمي،با +rم) موجها قدرات العقل في فهم وتحليل العمل الفني للوصول الى الهدف والمضمون الفكري لله.وأخيرا فإن المفاهيميه التنكيلية تعُني بالتعبير عن الأفكار والمعاني أكثر من اعتنائها بجمالية التعبير الفني

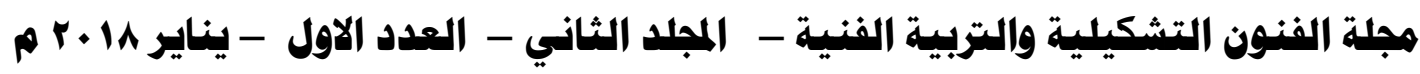




\section{التحملات المفاهيسية للفن التشكيلي السعودى المعاصي}

(القويضي، Y ( آم). فهو يتطلب التمعن في قراءة العمل والمعايشة المتلقي لمراحل الابداع والتأملالعمل وما يحمله من علامات وارشادات نساهم في إيضاح الهدف المفاهيمي

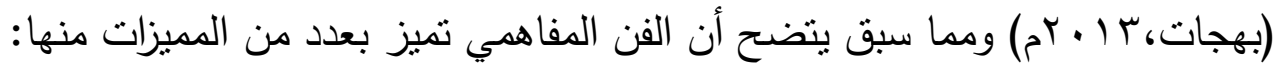

• الفن المفاهيمي هو حالة تحويل فكرة ما وجعلها ملموسة.

$$
\begin{aligned}
& \text { • هو فن حسسي وليس نظري. } \\
& \text { • بعد خروج عن الفن التقليدي. }
\end{aligned}
$$

• برتكز على الفكر الخالص بينما تقف العاطفة للتعبير على الجانب الاخر • • أنه يمثل مرحلة من النشاط ما بين الفكرة والنتاج النهائي.

• يطرح أسئلة عن طبيعة ما يفهم من الفنأن الفن وجد بالطبيعة كمفهوم.

$$
\text { • الفن مجال لتأمل العقلاني النقدي. }
$$

• بعتبر أعادة تعريف للفن بجوهره لا وظائفه ولا نفعية وهو مجرد من الغايات.

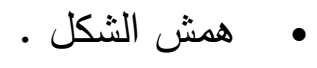

• الواقع بالنسبة للفن المفاهيمي هو المجال الاساسي لأي مقابلة جمالية.

$$
\text { • أن الفن المفاهيمي يصبح احيانا فيزيقيا (ماديا)تماما. }
$$

أن الفن المفاهيمي هوفن الأنماط الفكرية متضمنا أب وسائل براها الفنان مناسبة، ومن أوائل الفنانين الذي استخدموا المفهوم المفاهيمي في تتكيلاتهم التعبيرية الفنان"جوزيف كوسوت 


\section{التحولات المفاهيسية للفن التشكيلِي السعودى المعاصر}

(Joseph Kosut) الفتوغرافية .والتحديد اللغوي لكلمة كرسي •بهدف تمثنل الثنيء .ولما كان الثيء الحسي بعتبر بديهيا .فان ما يهمنا فيه هو ادراكه وفهمه .والكلام عنه يأخذ مكان الفن عندما يعبر عن بعده الجمالي بحرية حيث توجد شخصية الثيء المستخدم في الصورة بين هذه الثناثة، هل هي في الثيء نفسة ،أم فيما يمثلك أم في الوصف الكتابي أو الثفوي، واذا كان من المكن اكتثاف هذه الثخصية في الاثشياء الثلاثة.

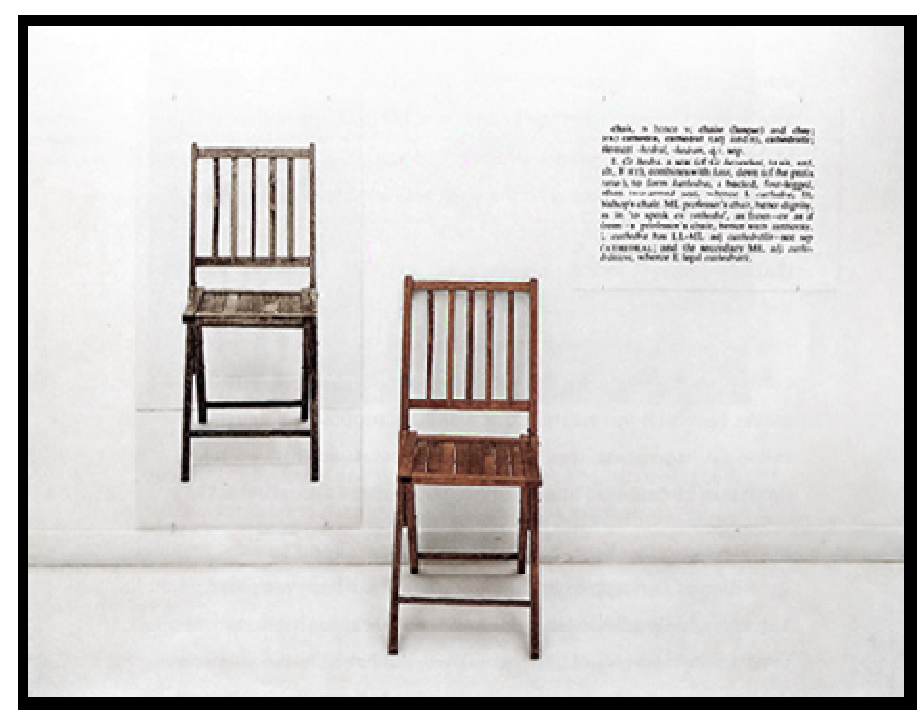

Joseph Kosuth, One two Three chairs, 1964

http://www.institut-numerique.org

هجلة الفنون التشكيلية والتربية الفنية - الجلد الثاني - العدد الاول - يناير Al م م A 
كما تتضح المفهوم التشكيلي للفن الدفاهيمي في عمل الفنان دانيل بورين( Daniel (Burren عبر الفراغ المتاح في قاعدتين عرض مفتوحتين فيما بينها.

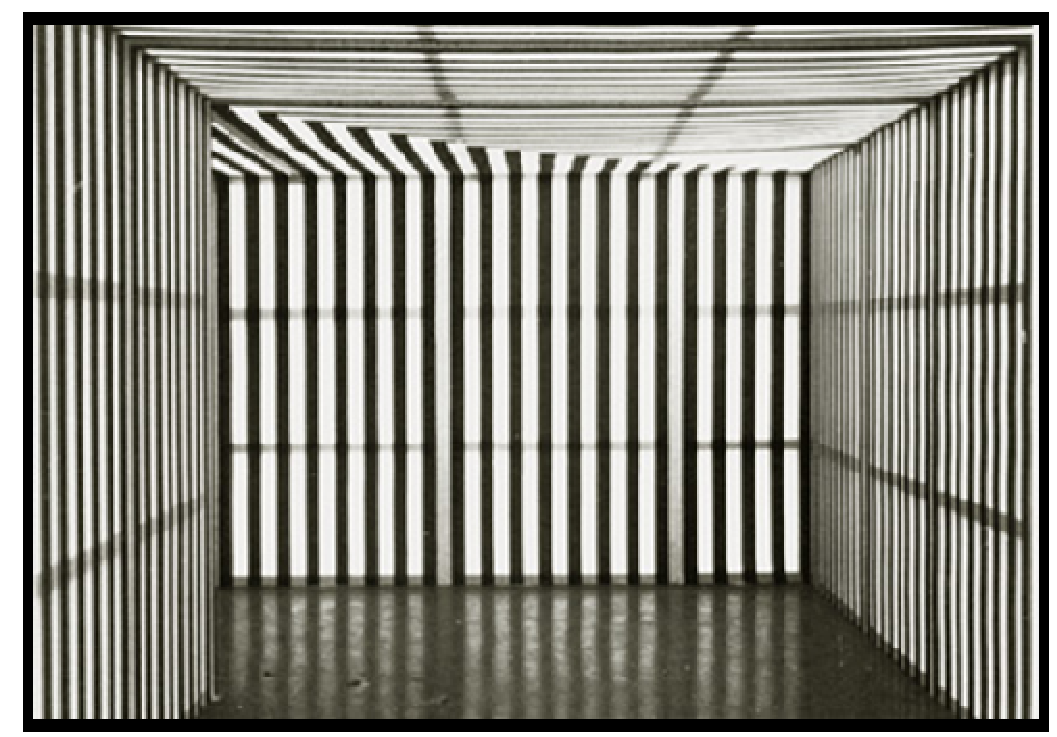

وينتقل التزكيز من الثيء الى الفكرة بأسلوب مختلف في عمل جون بورفسكي (John Borowski قصد مشيرا الى الزوال ، وأنتجه الفنان ليناسب فراغ معين ومناسبة معينة.

هجلة الفنون التشكيلية والتربية الفنية - الجلد الثاني - العدد الاول - يناير Al م م A 101 


\section{التهولات المفاهيسيـية للفن التشكيلي السعودى المعاصر}

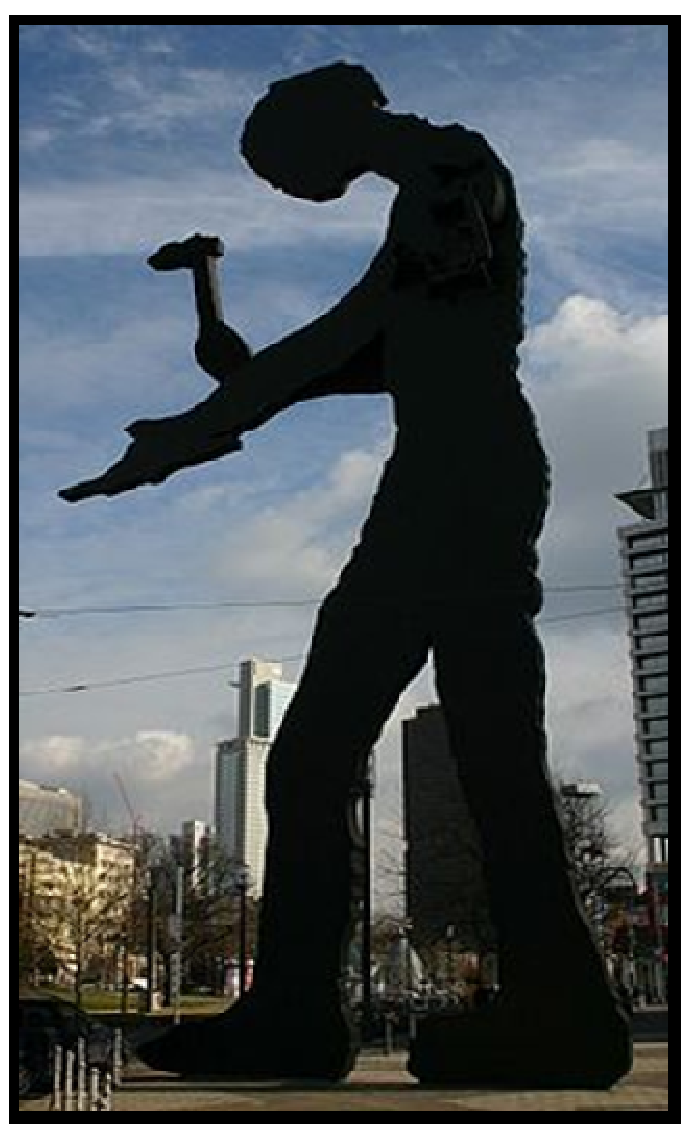

design-milk.com

المحور الثاني: المضمون المفاهيمي في الفن التثكيلي السعودي:

لقد أبرز الفنان التشكيلي السعودي من خلال أعماله الفنية المعاصرة بجماليات التعبير

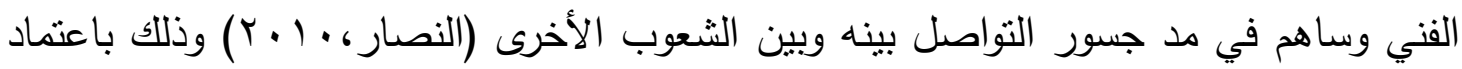

على الأساليب الفنية العالمية المعاصرة منها الفن المفاهيمي بأنواعه التشكيلية.

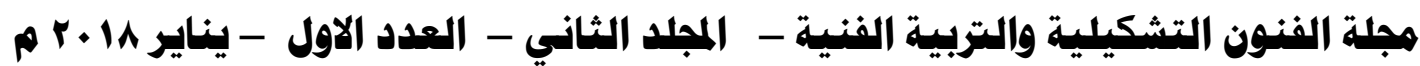




\section{التحولات المفاهيسية للفن التشكيلِي السعودى المعاصر}

لقد مر الأسلوب الدفاهيمي في الفن السعودية بمراحل من التطور ليصل في نهاية المطاف الى إنجازات تشكليه عالمية جذبت العديد من المهتمين والنقاد. حيث أصبح المضمونالتشكيلي للفن المفاهيمي السعودييقوم على" المقابلات الثنائية المتضادة مثل: الثكل/المضمون، الطبيعة/الثقافة، الفكر/الحس، الواقع الخيال، الدال/الددلول" (عطية 11 ـ بام، ص Y IVT).فقد هيمنة المنضادات التشكيلية على العمل الفنيموظفا كل عنصر وتقنية لتكون مكمله للأخرى، مكونا طاقة تعبيرية في العمل التشكيلي وكان هذا الأسلوب مبسط في بدايات الاعمال المفاهيمي للفنان السعودي المعاصر وتطور تدريجا ليصل الى مرحلة الابداع التشكيلي

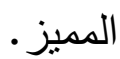

وقد اعتمد التحليل الفني التتكيلي الدفاهيمي على ما تحمله تتكيلاته التعبيرية من دلالات ومضامين تدعو المتذوق الى استحضار دلالتها الفكرية والفلسفية والاجتماعية والسياسية والدنية.(عطية، (1 • rم). فالفن الدفاهيم السعودي أرتبط بالتيارات الفنية المختلفة التي ظهرت في العالم الفني بأسلوب متميز عن غيرها فكانت تشكيلات الفنانين السعوديين تتميز بطابع جمالي خاص بها بالرغم من الاندماج الحضاري والتقافي بين شعوب العالم. وقد أستخدم الفنان التشكيلي السعودي المفهوم الفلسفي في التعبيرات الفنية لإعماله الدفاهيمه دامجا إياها بأساليب فنية منتوعة ومتمايزة من ناحية المضمون والفكرة للوصول الى المعنى والهدف. 


\section{التحولات المفاهيسيةية للفن التشكيلي السعودى المعاصر}

وقد كانت الفنون السعودية تتماشى مع مسارات فنون العالم بالرغم من التمايز الثقافي والاجتماعي في بادئ الامر، ولكن مع تقدم التقنيات ووسائل التواصل الاجتماعي اندمت تشكيلات الفنون السعودية المعاصرة بفنون العالم بالرغم من احتفاظها بميز تشكيلة تتفرد بها ويمكن من خلالها التعرف على أن هذا العمل يرجع لاحد الفنانين السعوديين لما تربط به مضامينها الفكرية بأسلوب الحياة المعاثده وثقافة المجتمع الدينة والمعرفية والثعبية. ومن المسارات الفنية للتشكيل السعودي المعاصر جماعة( شتا) التي عنيت بالفنون المعاصرة باحثة عن انطلاقة جديدة للفن السعودي مغاير ومختلفة عما كان علية في زمن الرواد " جاءت شتا لتعبر عن مفاهيم جديدة منصلة بالحياة، في عالم يضج بالأسئلة والتغيرات، وفي مجتمعات ملئ بنقافات مختلفة وبقضايا جوهرية تتصل بالمكان والزمان. لكنها لا تلبث أن تجتمع في آفاق إنسانية مشتركة، مستقيدة من مفاهيم غير محددة، كالمجانية والمصادفة في العمل الفني، والتأسيس من الفراغ والأدائية الجديدة" (موقع الرصيف،11.07.2016 ). ومن التيارات الفنية نجد(الحروفية) التي أعتمده فنانيهاعلى تشكيلات الخط العربي كأداة للتعبير عن الدفاهيم الفكرية بممارسه غاية الحرف الأصيل، الذي يعانق التعبير الفني ، بأسلوب تصاعدي تتكيلي ينقل الهنلقي الى عالم التأمل الفكري الاصيل، بتتقل بين الحروف، للوصول الى الهذف من المعنى المطروف للفكرة. 


\section{التحولات المفاهيسيةية للفن التشكيلي السعودى المعاصر}

لقد أصبح لكل فن تشكيلي لغة تخاطب نساهم في تكوين مفهوم اجتماعيونقافي

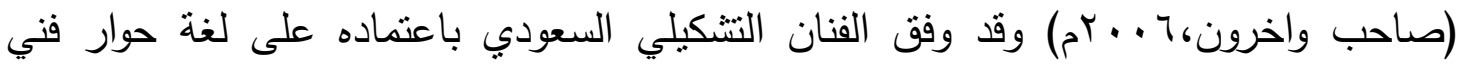
منوافق مع مبادئ ومفاهيم المجتمع السعودي بصفه خاصة والدجمع العالمي بصفة عالمه ومحملة برسائل تربوية وسلوكية وفكرية موجهه للمتلقي. استهدفت البناء القيم للإنسانية.

أن الفن المفاهيميهو " الحيز الفكري الاحتجاجي الذي يبدعه الفنان، لكي يحقق من

خلاله منطلقين، المنطلق المادي والروحي" (رضا،ه ـ . بم، صـ/1) في العمل الفني بكون وسيلة أتصال وتواصل بين الفنان والمتلقي بين ما تتطلبه التقنية الفنبة وما تحمله من مضامين فكرية تحقق الجمال التكويني في نفس المتلقي وهذا ما نجدة بالتتكيلات الفنية المفاهيمة لدى الفنان

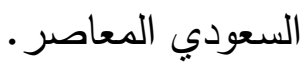

المحور الثالث: التحولات المفاهيمة في الفن التشكيلي السعودي المعاصر.

أن الفن" عنصر ينقل الثقافة بجانبيها المادي والمعنوي، وعاملا من عوامل البناء

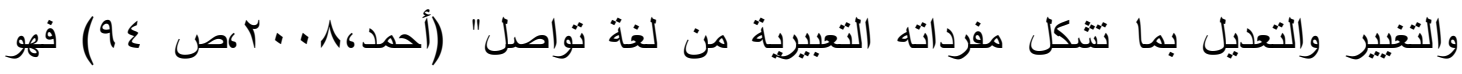
حصيلة جهود الفنان الفكرية والتقنية والتعبيرية التي يقدمها للمتلقي بهدف أيصال فكرة وهدف محدد عند التذوق الفني مطوعا موضوع العمل الفني وفضائه التتكيلي لهذا المفهوم.

أن للتفاعل التقافي الأثر الواضح في التحولات المفاهيمية للتصوير التشكيلي السعودي المعاصر ، إلا أن الفنان السعودي وصل الى التميز التتكيلي في التعبيرالفني بعدما مر بعلاقات

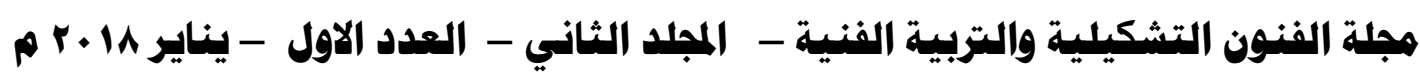




\section{التحولات المفاهيسية للفن التشكيلي السعودى المعاصر}

متتوعة ومتداخلة أدت الى تغير الأنماط والأساليب الثقافية للمجتمع (وهبة،م . ب السعودي ساهمت في ظهور التحولات المفاهيمة في التشكيلي الفني.

كانت بدايات ظهور مبادئ التحولات الدفاهيمه للفن التشكيلي في المملكة العربية

السعودية على يد كلا من صفية بنت زقر وسعيد العبيد، ومحمد الصندل ومنصور كردي وغيرهم

(السليمان، ... بم) حيث جمعوا بين المفاهيم الفكرية للتعبير الفني والموضوع التتكيلي باحثين عن علاقات تربط بين الثقافة العالمية للفنون وتحولاتها الفكرية العالمية وأيديولوجية المجتمع السعودي المرتبطة بالإرث الثقافي والديني والاجتماعي للمجتم. معتمدين على أن الإدراكالحسي للفنون مركب يساهم في اندماج المجال للافتراضي للعمل الفني بما يحمله من علاقة ذات معنى وهدفامع العالم المرئي ، وأن جذور هذه المعاني والأهداف للفكرية تقع وراء نطاق الوعي؛ بين حدودالقوة التقافية المتشكلة من الروية البصرية والثعور الحسي الداخلي الذي يظهر في مرحلة الاندماج الفني ، ومراعين لما لزمن من فضاء موضوعي يرتبط بمكان وموضوع العمل، للوصول الى إدراك جوهري لفكرة العمل التشكيلي المتعلق بصميم إحساسنا الجمالي للعمل الفني بما يحمله من الثقل والتنوازن ، والحركة والثبات، والوحدة والعمق للتعبير الفنيموجها المتلقي للهدف الفكري من العمل الفني. حيث أصبح التحليل التشكيلي في الفنون التتكيلةالسعودية المعاصرةلا تعتمد على الثكل فقط وإنما أرتبط بالموضوع التعبيري والعناصر والخامات واللون والإضاءة

$$
\text { وعلاقاتها ببعضها وما تقدمة من أنظمة ودلالات (صاحب واخرون، ج . . م) }
$$

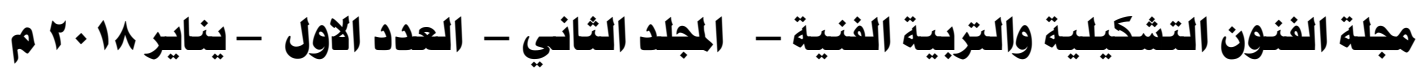




\section{التحولات المفاهيسية للفن التشكيلِي السعودى المعاصر}

أن الفنان السعودي وقف أمام المفهوم الفلسفي للفن المفاهيمي موقف الباحث عن الهوية

المتأصلة بالثقافة البصرية وما تحمله من مدلولات فكرية واجتماعية. فالهوية كانت من أبرز ما ساهم في تحول الفن الدفاهيمي السعودي من فن غير مقبول الى فن عالمي حيث توصل الفنان السعودي المعاصر الى نتيجة معرفية تعتمد على أسس الهوية الدينة وما تحمله من ثأثنرات ثقافية واجتماعية وسياسة. وهذا ما ساهم في تطوير المجتمع ودمجه بالمجتمع العالمي. فالهوية لدى الفنان السعودي هي ذات بعد داخلي يساهم في تعدد الرؤى الفكرية للموضوع الواحد. فالفن التتكيلي السعودي المعاصر وصل الى تميز فني في مجال الفن الدفاهيمي من خلال عدد من التحولات منها فكرية وأخرى اجتماعية وثقافية وسياسية. فالفنان السعودي شارك في العديد من المعارض العالمية ناقلا تشكيلاته التعبيرية المرنبطة بواقعه المعاش للعالم. وذللك بما تحمله أعماله الفنية من مضمون فكري يرتبط بالمجتمع السعودي والعالمي في نفس الوقت. فالتقنية الفنية المستخدم أرتبط بالإرث الثعبي السعودي والفكر الديني للمجتمع مطوعا إياها بأسلوب معاصر جذبت المتلقي من شتى بقاع الأرض.

أن الفنان السعودي المعاصر أنتقل من كونه عامل حرفي أو ناسخ فني أو مصور فوتوغرافي الى فنان تشكيلي مميز طوع الخامة والتقنية والاداة لهدف محدد من العمل الفني وهي الفكرة. جامع بين الثقافة العالمية وتحولات العصر المختلفة والثقافة المحلية بما تحمله من جذور شعبية ودينه، معرفا العالم على مفاهيم اجتماعية فكرية وفلسفية للمتمع السعودي ومساهما في تطور العلاقات المعرفية من خلال الحوار الحضاري بين فنون العالم والمتلقي، فأصبح يعتمد

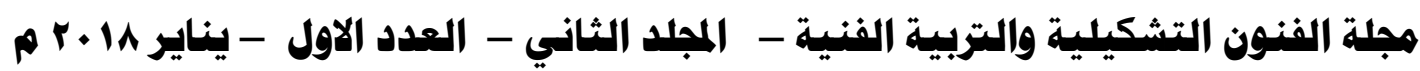




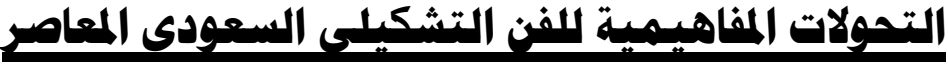

على الحوارات البصرية للموضوع الفكري التي لا تعتمد على شكل بصري واحد وانما أعتمد على الروئية البصرية الواحه للمفهوم الفكري بتعدد التنكيل البصري.

نرى هذه المفاهيم تتجسد في تجربة|لفنان عبدالناصر غارم بطرح تساؤل عن ماهية

الطبيعة؟ وذلك من خلال مقاربة الطبيعة بوجودنا الإنساني، وفي عمل فيديو فني Video Art، تحت عنوان "الصراط"، الذي يطرح سؤالاً مصيرياً مستلاً من وقائع الحياة المعاش في المجتمع المسلم والمصير النهائي للأنسان. وتللك القراءة الجديدة للموروث الثقافي الديني في عمله "القبة هذا التتكيل الفني المحمل بفلسفة المجتمع الدينية وأهدافه الفكرية التي تعظم مكانة الثعائر الدينة بما يحمل العمل من مساحة وتقنية والوان المعبر عن فكرة تعظيم المكانة الاجتماعية للمصلي وتعبيرا عن جمالية الحس الروحي له.

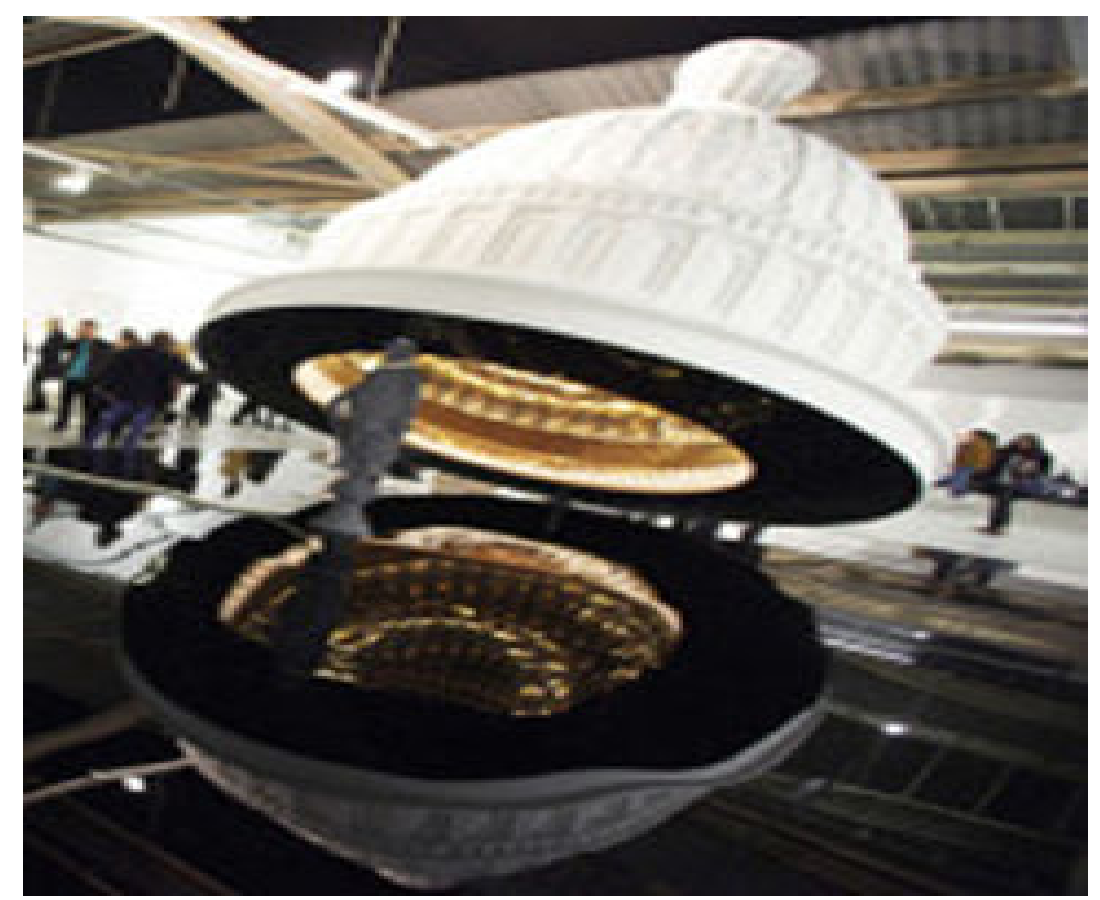

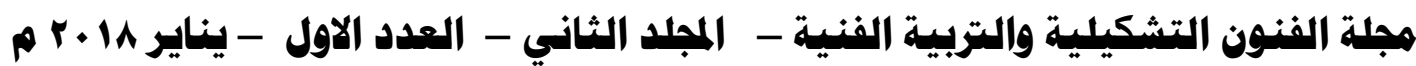




\section{التدولات المفاهيسيمية للفن التشكبلِي السعودى المعاصر}

والفنان" أحمد ماطر قدم عدد من الاعمال التشكيلية المفاهيمةالتي نوضح مجال

التحولات الفكرية للفن المفاهيمي منهاما أعتمد فيها علبالمؤثرة كالمغناطيس بعملة المسمى "مغناطيسية" المتصل الذي يحمل مدلولا فكريا متأصل في الدجتمع السعودي والثعائر الدينية في تلخيصه لفريضة الحج من خلال تجسيد بيت اله الحرام مستلاهما ذلك الإحساس النفسي الذي يجذب المسلمين للكعبة المشرفة.

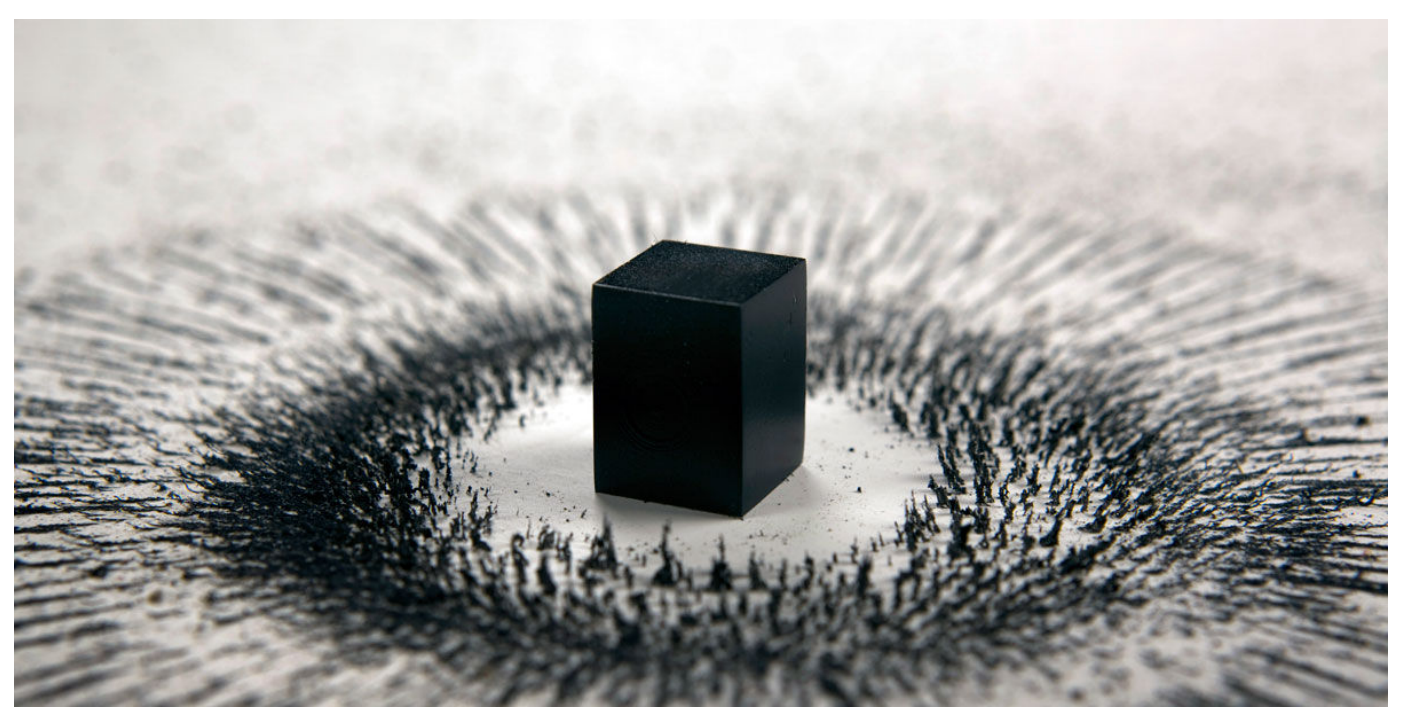

هجلة الفنون التشكيلية والتربية الفنية - الجلد الثاني - العدد الاول - يناير Al م م A 


\section{التحولات المفاهيسيـية للفن التشكيلي السعودي المعاصر}

كما تميزت الفنانة "منال الضويان "في طرحها لقضايا المرأة ضمن حساسية جديدة في المجال الدفاهيمي. تلك القضايا المتصلة بالسفر والعادات والثقاليد، ومساحات التعبير والعمل، ومرتبطة باللغة والصورة والمفارقات التي تتولد منهما موجهة نظر العالم لما يقيد المرأة السعودية ويمنعها من التميز والدخول في مجلات مختلف تأثر في التحولات الفكرية. وكان لهذا العمل الأثر الكبير لدى الأوساط النقافية في المجتمعات المختلفة.

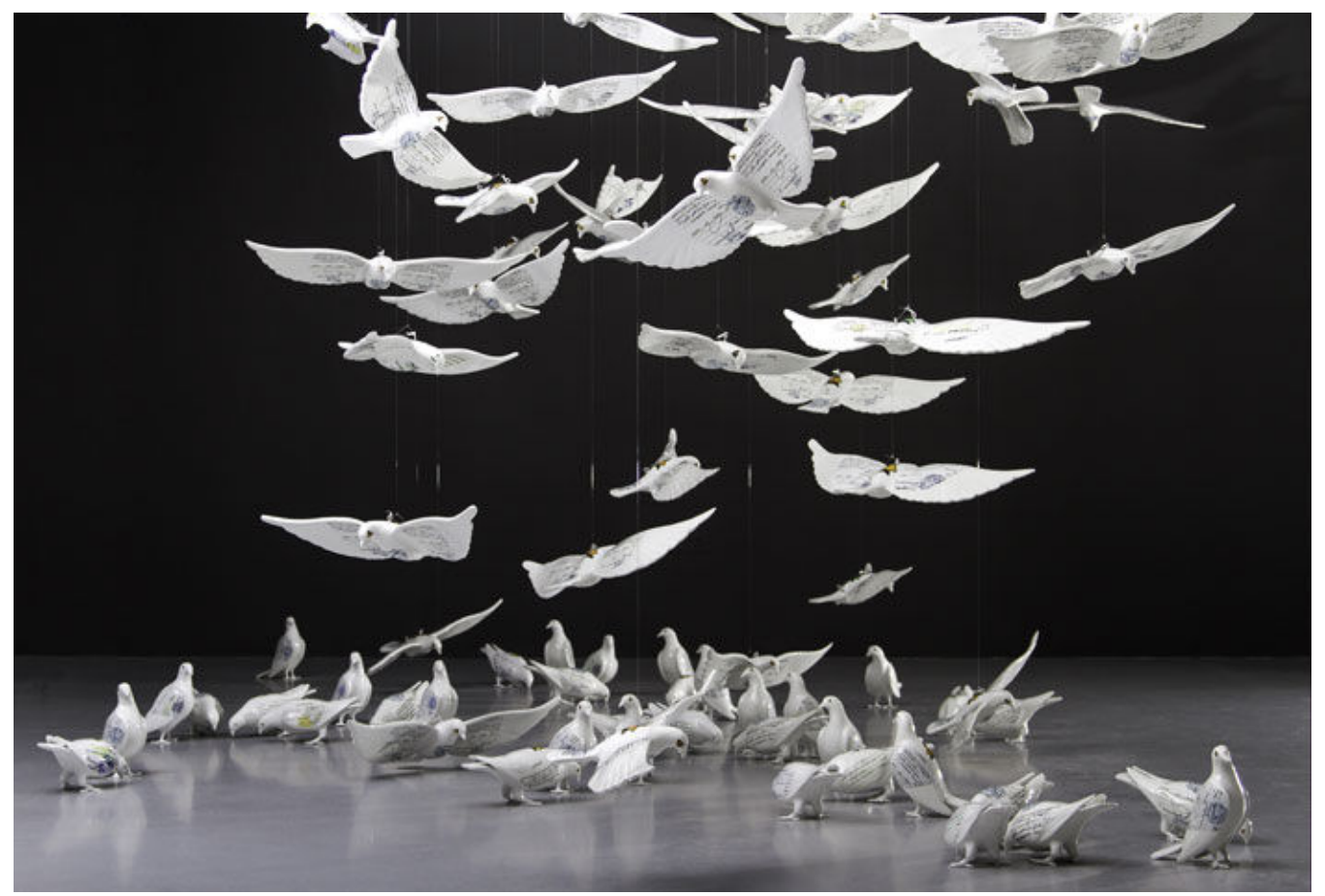

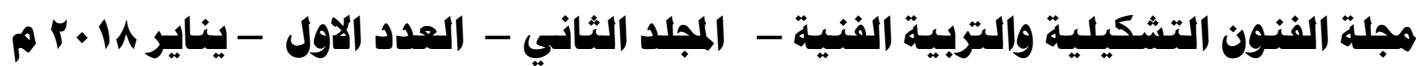




\section{التحولات المفاهيسية للفن التشكبدي السعودى المعاصر}

ومن الفنون التشكيلية المفاهيمية المعاصر عمل الفنان السعودي (صديق واصل)الذي

لقب بالنحات الفيلسوف بعمله الذي عمد فيه الى التعبير التأملي العقلي للتنلقي للوصول الى تهاللك المصير وتتابع الاعمال اليومية مشكلا مشكلة فكرية تدعو المتلقي للبحث الدلالي للوصول

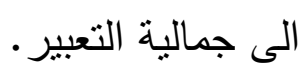

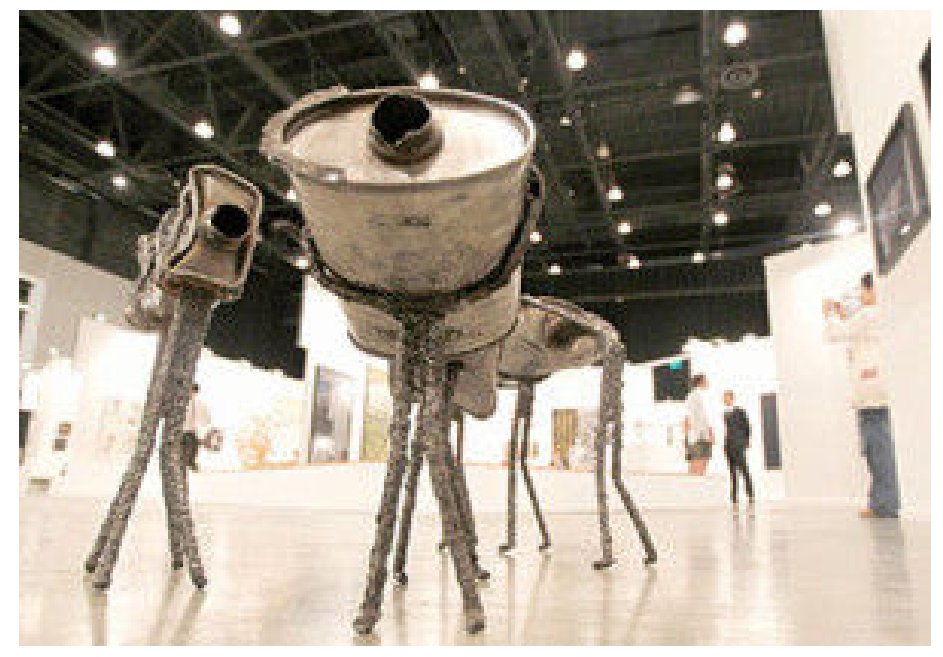

ونجد عمل الفنانة دانه الثطيريبعنوان " خط الزمن" الذي عبرت به عن التتابع الفلسفي الفكري الذي يهدف الي تدعيم مفاهيم الامتزاج الحضاري رغم التتوع وأن هذا الامتزاج متتاعم وجمالي داعية المتلقي الى التمعن الاندماج الفني بين التقنية الخامة للعمل الفني. 


\section{التحولات المفاهيـيـية للفن التشكيلي السعودى المعاصر}

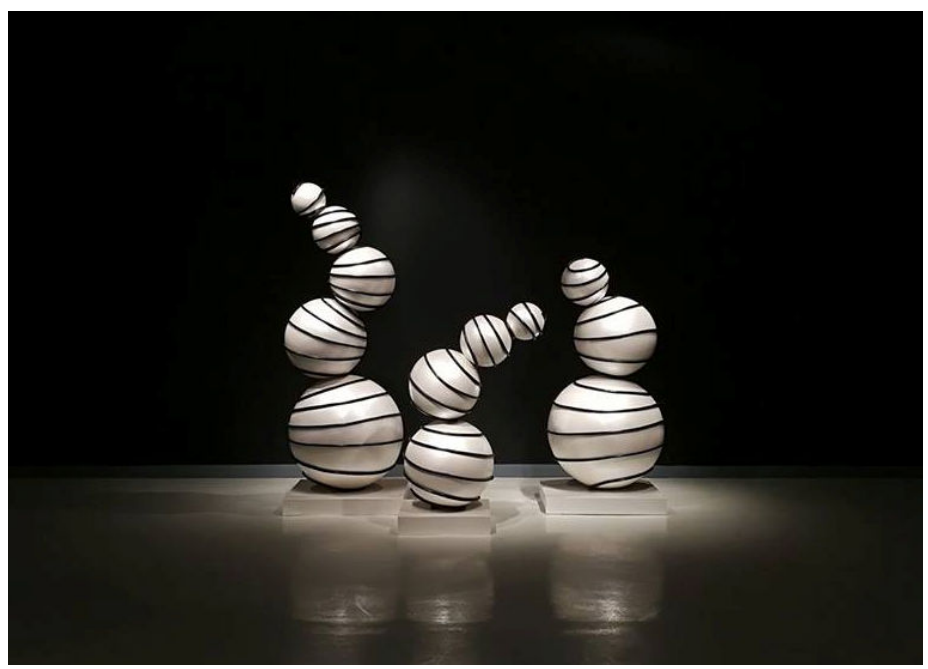

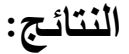

جاءت النتائج وفقا للاراسة التحليلية للبحث على النحو التالي:

• للفن المفاهيمي المعاصر مضمون فكري عالمي أتبع الفنانين على اختلاف

$$
\text { مجتمعاتهم. }
$$

• أن المضمون الفلسفي للفن المفاهيم في الفنون النتكيلية المعاصرة تميز بارتباطه

بالعلاقات التبادلية بين الموضوع التعبيري والثقافي الاجتماعية والدينة والسياسية.

• مر الفن المفاهيمي في الفنون النتكيلية السعودية المعاصرة بتحولات مفاهيمه ساهمت

$$
\text { بوصول الفن المفاهيمي السعودي الى العالمية. }
$$

• من أبرز التحولات المفاهيميه للفنون التشكيلي المعاصرة نقل الإرث الثعبي والديني

$$
\text { للمجتمع السعودي الى العالم. }
$$

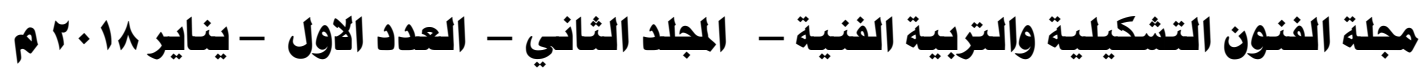


عقد ورش عمل ومحاضرات لمناقثة التوجهات الحديثة في المجال التنكيلي في

$$
\text { الفنون السعودية المعاصرة. }
$$

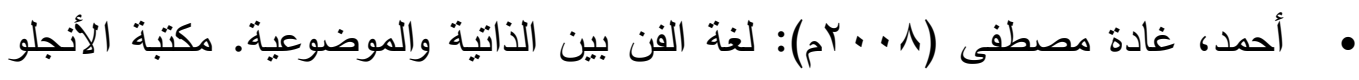

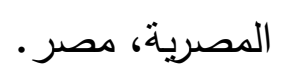

• أمهز ، محمود(1997 (1):التبارات الفنية المعاصرة، شركة المطبوعات للتوزيع، بيروت.

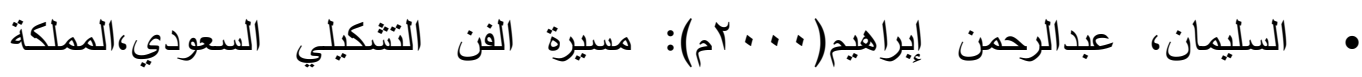

$$
\text { العربية السعودية، وزارة الثقافة والاعلام،ط؟. }
$$

• القويضي، يسري(r ( • rم): المفاهيميه في التنكيل العربي -إيزيسلل إبداع والثقافة -

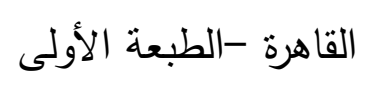

• النصار، سمر(• ( • (r)): مسيرة الحركة الفنية التشكيلية السعودية النشأة والتطور •

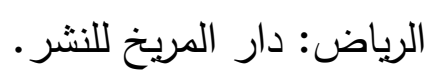

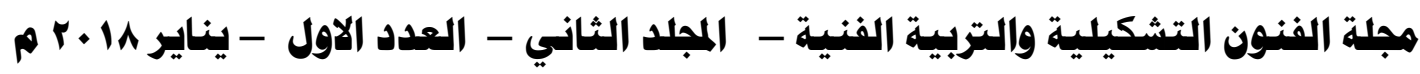




\section{التحولات المفاهيسية للفن التشكيلي السعودى المعاصر}

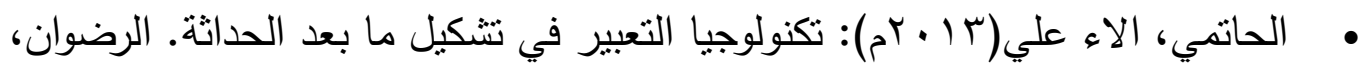

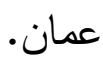

• آل وادي، شناوه و عبادي، رحاب(1) • (1): استطيقا المهش في فن ما بعد الحداثة كدار الصفاء للنشر والتوزيع، عمان.

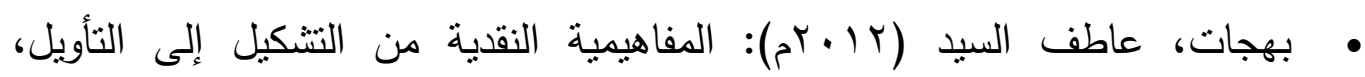
علامات في النقا الأدبي -النادي الأدبي الثقافي بجدة - السعودية، العدد 77 ،محرم

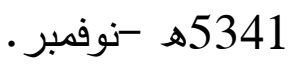
• خضر، محمد (11.07.2016) موقع الرصيف مقالة بعنوان: الله، التراث، الاستهلاك... قضايا الفن المفاهيمي السعودي. • سميث، ادورد لويس(بدون):الحركات الفنية منذه 9 (،وزارة الثقافة والاعلام،الثنارقة.

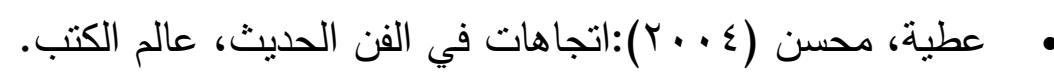
• عطيه، محسن محمد (ع . ㄷم): اتجاهات في الفن الحديث. القاهرة، عالم الكتب.

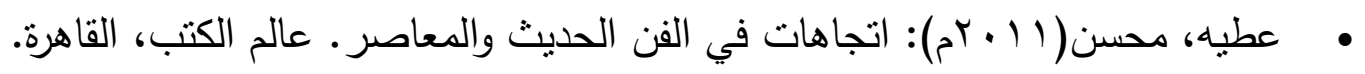
• صاحب، زهير وأخرون († . ⼴م): دراسات في الفن والجمال. مكتبة المجدلاوي،

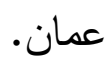




\section{التحملات المفاهيسية للفن التشكيلي السعودى المعاصر}

• قاسم، ولاء إبراهيم(1 (1 +r): فلسفة الحضارات وأثزها في الفن المعاصر كمخل لتدريس التصوير في كلية التربية النوعية في الإسكندرية. رسالة دكتوراه غير منشورة، قسم رسم وتصوير ، كلية التربية، جامعة حلوان.

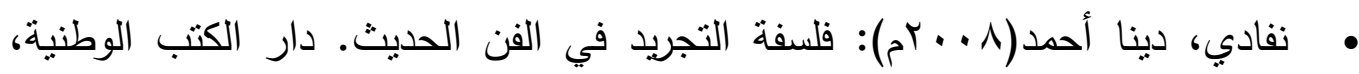
ليبيا. • وهبة، رباب علي(^ . • rم): تفاعل نقافات متتوعة في تتكيل القيم الجمالية للفن المصري المعاصر • رسالة دكتوراه غير منشورة، قسمنقد وتذوق، كلية التربية، جامعة حلوان.

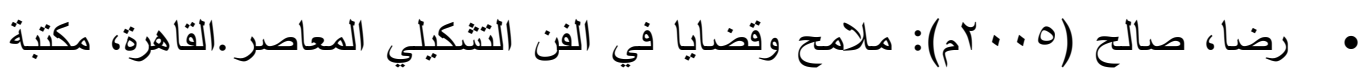
الأسرة. 
ملخص البحث:

تتاول البحث الحال الدفهوم الدفاهيمي للفنون العالمية ومنها الفن الدفاهيمي السعودي المعاصر، وذلك بمحاولة الإجابة عن مشكلة الرئيسة وهي ما التحولات المفاهمية لدى الفنان التنكيلي السعودي المعاصر . حيث فهدف الدراسية الى التعرف على المضمون المفاهيمي في الفنون التشكيلية المعاصرة. والتأكيد على المضمون المفاهيمي في الفنون التنكيلة السعودية المعاصرة. وصولا للكثف عن التحولات المفاهية في الفنون التشكيلة لسعودي المعاصرة. متبعة المنهج الوصفي التحليلي في وصف مفهوم الفن الدفاهيمي، وتحليل نماذج من الاعمال المفاهيمية السعودية. وقد توصلت الدراسة الى عدد من النتائج وهي أن للفن المفاهيمي المعاصر مضمون فكري عالمي أنبع الفنانين على اختلاف مجتمعاتهم. وأن المضمون الفلسفي للفن المفاهيم في الفنون التثكيلية المعاصرة تميز بارتباطه بالعلاقات التبادلية بين الموضوع التعبيري والثقافي الاجتماعية والدينة والسياسية. لقد مر الفن المفاهيمي في الفنون التشكيلية السعودية المعاصرة بتحولات مفاهيمه ساهمت بوصول الفنالمفاهيمي السعودي الى العالمية، ومن من أبرز التحولات المفاهيميه للفنون التشكيلي المعاصرة نقل الإرث الثعبي والديني للمتمع السعودي الى العالم. وتوصي الدراسة بضرورة الاهتمام بتأصيل الفنون التتكيلية السعودية المعاصرة. وتدعيم التوجهات الفكرية المعاصرة في الفنون التنكيلة السعودية.

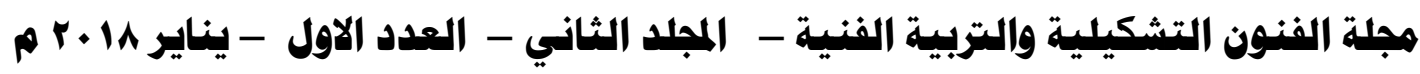


:Abstract

The research dealt with the conceptual concept of the world arts, including contemporary Saudi conceptual art, by trying to answer themain question, What are the conceptual transformations of the Saudi artist?. Where the objective of the study to identify the conceptual content in contemporary conceptual arts. Emphasis on conceptual content in the contemporary Saudi arts. And to uncover the conceptual transformations in the contemporary Saudi arts. Following the analytical descriptive approach in describing the concept of conceptual art, and analyzing examples of Saudi conceptual works. The study found a number of results: that contemporary conceptual art has a global intellectual content that followed artists from different societies. And that the philosophical content of art concepts in contemporary conceptual arts is characterized by its relationship to the interrelationships between the expressionist and cultural, social, urban and political. The conceptual art in the contemporary Saudi conceptual هبلة الفنون التشكيلية والتربية الفنية - المبلد الثاني - العدد الاول - يناير ش1 بم مي 
arts has undergone transformations of concepts that contributed to the arrival of Saudi conceptual art to the world, and one of the most significant conceptual transformations of modern conceptual art is the transfer of the popular and religious heritage of the Saudi society to the world. The study recommends that attention should be given to rooting contemporary Saudi conceptual arts. And strengthening contemporary intellectual trends in the Saudi arts.

Keywords: conceptual art, contemporary art, Art Appreciation and criticism 